\title{
On Factor Decomposition of an Ergodic Groupoid
}

\author{
By \\ Shigeru YAMAGAMI* \\ Factor decomposition of cocycle regular representations of ergodic groupoids whose \\ stabilizer groups are uniformly abelian is studied.
}

\section{Introduction}

Given a measure groupoid $\Gamma$ and a torus-valued 2-cocycle $c$, a Hilbert algebra $\mathfrak{A}_{c}$ is defined in a definite way and the associated representation of $\mathfrak{A}_{c}$ on the $L^{2}$-completion of $\mathfrak{A}_{c}$ is called $c$-regular representation of $\Gamma$. In the study of $\mathfrak{A}_{c}$, one of the basic problems is the factor decomposition of $\mathfrak{A}_{c}$. Let us have a try at it in terms of decomposition of $\Gamma$. Since the ergodic decomposition of $\Gamma$ induces a central decomposition of $\mathfrak{A}_{c}$, the problem is reduced to the case when $\Gamma$ is ergodic. If, furthermore, $\Gamma$ is supposed to be a principal one, every $c$-regular representation of $\Gamma$ is known to generate a factor ([5]) and there remains no problem. In this paper, we deal with groupoids whose stabilizer groups are uniformly chosen (see the beginning of $\S 1$ for the precise meaning) and investigate the above mentioned problem.

Organization is as follows: $\S 1$ gathers facts needed in later sections. In $\S 2$, we construct a measure space $S^{*}$ with an equivalence relation, from the information of $(\Gamma, c)$, and show that ergodic decomposition of $S^{*}$ is equivalent to the factor decomposition of $\mathfrak{A}_{c}$ in $\S 3$. In $\S 4$, we select a subgroup $\Sigma$ of the stabilizer of $\Gamma$ and ergodic quotient of $S^{*}$ is identified with a certain $\hat{\Sigma}$-principal homogeneous space. $\S 5$ is only a matter of formulation and gives a factor decomposition of $(\Gamma, c)$, i. e., factor component of $\mathfrak{A}_{c}$ is realized as a cocycle regular representation of the quotient groupoid $\Gamma / \Sigma$. In $\S 6$, an example is

Communicated by H. Araki, October 5, 1987.

* Department of Mathematics, College of General Education, Tohoku University, Sendai 980, Japan. 
discussed.

I am grateful to Professor Araki for valuable comments and to S. Kawakami for helpful discussion (in particular, arguments in the proof of Lemma 2.3 is owing to his suggestion).

\section{\&1. Preliminaries}

Let $\Gamma$ be an analytic groupoid (=Borel groupoid with its Borel structure analytic) and $G$ be a locally compact second countable abelian group. We assume the uniformity of stabilizer groups of $\Gamma$ in the following sense: For each $x \in \Gamma^{(0)}$, an isomorphism $\iota_{x}$ of $G$ onto $\Gamma_{x}^{x}(=\{\gamma \in \Gamma ; r(\gamma)=s(\gamma)=x\})$ is assigned and satisfies

$1^{\circ}$ for $\gamma \in \Gamma$ and $g \in G, \iota_{r(\gamma)}(g) \gamma=\gamma \iota_{s(\gamma)}(g)$

$2 G \times \Gamma \ni(g, \gamma) \mapsto \iota_{r(\gamma)}(g) \gamma$ is a Borel map.

For notational simplicity, we write $\iota_{r(\gamma)}(g) \gamma\left(\operatorname{resp} . \gamma \iota_{s(\gamma)}(g)\right)$ as $g \gamma$ (resp. $\gamma g$ ). We assume that $\Gamma$ has a faithful $\sigma$-finite transverse function $\left\{\nu^{x}\right\}_{x \in \Gamma^{(0)}}$ and a transverse measure is specified by a pair $(\mu, \nu)$ where $\mu$ is a $\sigma$-finite measure in $\Gamma^{(0)}$. Then $m(d \gamma)=\int \mu(d y) \int \nu^{y}(d \gamma)$ is a $\sigma$ finite measure in $\Gamma$ and quasi-invariant under inversion $\gamma \mapsto \gamma^{-1}$. The measure groupoid $(\Gamma, m)$ is called ergodic if for each saturated Borel set $B$ of either $\mu(B)=0$ or $\mu\left(\Gamma^{(0)} \backslash B\right)=0$ holds. In the following, $(\Gamma, m)$ is supposed to be ergodic. For a normalized $\boldsymbol{T}$-valued Borel 2-cocycle $c$ of $\Gamma$, let $\mathfrak{A}_{c}$ be the set of functions $\xi$ on $\Gamma$ satisfying (1) for each $y \in \Gamma^{(0)},\left.\xi\right|_{\Gamma^{y}}$ and $\left.\xi^{*}\right|_{\Gamma^{y}}$ belong to $L^{2}\left(\Gamma^{y}, \nu^{y}\right)$

(2) $\quad \xi$ and $\xi^{*}$ belong to $L^{2}(\Gamma, m)$

$$
\sup _{y \in \Gamma^{(0)}} \int \nu^{y}(d \gamma)|\xi(\gamma)|<+\infty \text { and } \sup _{y \in \Gamma^{(0)}} \int \nu^{y}(d \gamma)\left|\xi^{*}(\nu)\right|<+\infty
$$

where $\xi^{*}(\gamma)=\overline{\xi\left(\gamma^{-1}\right) c\left(\gamma^{-1}, \gamma\right)}$. We can define in $\mathfrak{A}_{c}$ a multiplication and an inner product as follows.

$$
\begin{aligned}
& \left(\xi_{1} \xi_{2}\right)(\gamma)=\int \nu^{r(\gamma)}\left(d \gamma^{\prime}\right) \xi_{1}\left(\gamma^{\prime}\right) \xi_{2}\left(\gamma^{\prime-1} \gamma\right) c\left(\gamma^{\prime}, \gamma^{\prime-1} \gamma\right) \\
& \left(\xi_{1} \mid \xi_{2}\right)=\int \mu(d y) \int \nu^{y}(d \gamma) \overline{\xi_{1}(\gamma)} \xi_{2}(\gamma) .
\end{aligned}
$$

Then, together with the above ${ }^{*}$-operation, $\mathfrak{U}_{c}$ becomes a right Hilbert algebra ([3], [5]). For $\varphi \in \mathfrak{A}_{c}$, we denote the right multiplication of $\varphi$ by $R(\varphi)$. Through the natural decomposition

$$
L^{2}(\Gamma, m) \cong \int_{\Gamma^{(0)}}^{\oplus} \mu(d x) L^{2}\left(\Gamma^{x}, \nu^{x}\right),
$$


$R(\varphi)$ is decomposed as $\int^{\oplus} \mu(d x) R^{x}(\varphi)$. Here $R^{x}(\varphi)$ is the right multiplication by $\varphi$ in $L^{2}\left(\Gamma^{x}, \nu^{x}\right)$. For later use, we give a characterization of the von Neumann algebra $\mathfrak{R}_{c}^{\prime}(=$ von Neumann algebra generated by $\left.R(\varphi), \varphi \in \mathfrak{A}_{c}\right)$. Let $\gamma \in \Gamma$ and define a unitary map $U_{c}(\gamma)$ of $L^{2}\left(\Gamma^{s(\gamma)}, \nu^{s(\gamma)}\right)$ onto $L^{2}\left(\Gamma^{r(r)}, \nu^{r(r)}\right)$ by

$$
\left(U_{c}(\gamma) \xi\right)\left(\gamma^{\prime}\right)=c\left(\gamma, \gamma^{-1} \gamma^{\prime}\right) \xi\left(\gamma^{-1} \gamma^{\prime}\right) .
$$

Lemma 1.1 ([5] Th.4.1). Let $T=\int^{\oplus} \mu(d y) T^{y}$ be a decomposable operator in $\int^{\oplus} \mu(d y) L^{2}\left(\Gamma^{y}, \nu^{y}\right)$. Then $T$ belongs to $\mathfrak{A}_{c}^{\prime}$ if and only if there exists a suitable choice of measurable field of operators $\left\{T^{y}\right\}_{y \in \Gamma^{(0)}}$ such that $U_{c}(\gamma) T^{s(\gamma)}=T^{r(\gamma)} U_{c}(\gamma)$ for all $\gamma \in \Gamma$.

For $g \in G$ and $y \in \Gamma^{(0)}$ unitary operator $U_{c}^{y}(g)$ is defined to be $U_{c}\left(\iota_{y}(g)\right)$. We have

$$
U_{c}^{y}\left(g_{1}\right) U_{c}^{y}\left(g_{2}\right)=c_{y}\left(g_{1}, g_{2}\right) U_{c}^{y}\left(g_{1} g_{2}\right), \text { for } g_{1}, g_{2} \in G,
$$

where $c_{y}$ is a 2-cocycle of $G$ given by $c_{y}\left(g_{1}, g_{2}\right)=c\left(\iota_{y}\left(g_{1}\right), \iota_{y}\left(g_{2}\right)\right)$.

Lemma 1.2 ([3] Prop. 15). We can choose a sequence $\left\{\xi_{n}\right\}_{n \geq 1}$ in $\mathfrak{A}_{c}$ such that

(i) $\left\{R\left(\xi_{n}\right)\right\}_{n \geq 1}$ generates $\mathfrak{H}_{c}^{\prime}$,

(ii) for each $y \in \Gamma^{(0)},\left\{R^{y}\left(\xi_{n}\right)\right\}_{n \geq 1}$ generates $U_{c}^{y}(G)^{\prime}$.

Now we describe a factor decomposition of $U_{c}^{y}$. The following is implicitly contained in [2], [7]. Let $S(y)$ be a closed subgroup of $G$ defined by

$$
S(y)=\left\{g \in G ; c_{y}\left(g, g^{\prime}\right)=c_{y}\left(g^{\prime}, g\right) \text { for all } g^{\prime} \in G\right\},
$$

and set

$$
\begin{aligned}
& S^{*}(y)=\{b ; b \text { is a } T \text {-valued Borel function on } S(y) \text { satisfying } \\
& \left.b\left(g_{1}\right) b\left(g_{2}\right) b\left(g_{1} g_{2}\right)^{-1}=c_{y}\left(g_{1}, g_{2}\right) \text { for } g_{1}, g_{2} \in S(y)\right\} .
\end{aligned}
$$

Then $S^{*}(y) \neq \emptyset$ (symmetric cocycle is trivial) and, by point-wise multiplication, $S(y)^{\wedge}$ (=dual groups of $S(y)$ ) acts on $S^{*}(y)$ freely and transitively, so $S^{*}(y)$ inherits a standard Borel structure and an $S(y)^{\wedge}$-invariant measure $d b$ from $S(y)^{\wedge}$. For $b \in S^{*}(y)$, consider a Borel function $\xi$ on $\Gamma^{y}$ such that

(10) $\xi(g \gamma)=b(g)^{-1} c(g, \gamma) \xi(\gamma)$ for $g \in S(y), \gamma \in \Gamma^{y}\left(c(g, \gamma) \equiv c\left(\iota_{y}(g), \gamma\right)\right)$. 
Then $|\xi(\gamma)|^{2}$ defines a Borel function on $S(y) \backslash \Gamma^{y}$. Given a Haar measure $d s$ of $S(y)$, we can define a measure $\oint_{S(y) \backslash \Gamma^{y}} \tilde{\nu}^{y}(d r)$ in $S(y) \backslash \Gamma^{y}$ by the relation

$$
\int_{\Gamma^{y}} \nu^{y}(d \gamma) f(\gamma)=\oint_{S(y) \backslash \Gamma^{y}} \tilde{\nu}^{y}(d \gamma) \int_{S(y)} d s f(s \gamma)
$$

where $f$ is a positive Borel function on $\Gamma^{y}$. We impose on $\xi$ the following condition

$$
\oint_{S(y) \backslash \Gamma^{\eta^{\nu}}} \tilde{\nu}^{y}(d \gamma)|\xi(\gamma)|^{2}<+\infty .
$$

If $\mathfrak{S}_{b}$ is the set of all such $\xi^{\prime} s$, it is a Hilbert space by the following inner product.

$$
\left(\xi_{1} \mid \xi_{2}\right)=\oint_{S(y) \backslash \Gamma^{y}} \tilde{\nu}^{y}(d \gamma) \overline{\xi_{1}(\gamma) \xi_{2}}(\gamma), \xi_{1}, \xi_{2} \in \xi_{b 。}
$$

Let $\xi \in L^{2}\left(\Gamma^{y}, \nu^{y}\right) \cap L^{1}\left(\Gamma^{y}, \nu^{y}\right)$, then

$$
\xi_{b}(\gamma)=\int_{s(y)} d s b(s) c(s, \gamma)^{-1} \xi(s \gamma)
$$

defines an element in $\mathfrak{S}_{b}$. Taking various $\xi \in L^{2} \cap L^{1},\left\{\xi_{b}\right\}_{b \in S^{*}(y)}$ determines a measurable field structure for $\left\{\mathfrak{S}_{b}\right\}_{b \in S^{*}(y)}$. The correspondence $\xi \mapsto \int_{S^{*}(y)}^{\oplus} d b \xi_{b}$ gives rise to a unitary map from $L^{2}\left(\Gamma^{y}, \nu^{y}\right)$ to $\int_{S^{*}(y)}^{\oplus} d b \mathfrak{S}_{b}$, and under this isomorphism $U_{c}^{y}(g)(g \in G)$ is decomposed as $\int_{S^{*}(y)}^{\oplus} d b U_{c}^{b}(g)$. Here $U_{c}^{b}(g)$ is a unitary operator in $\mathfrak{S}_{b}$ and defined by

$$
\left(U_{c}^{b}(g) \xi\right)(\gamma)=c\left(g, g^{-1} \gamma\right) \xi\left(g^{-1} \gamma\right), \xi \in \mathscr{S}_{b} .
$$

Lemma 1.3 ([2], [7]).

(i) For $b \in S^{*}(y), U_{c}^{b}(G)^{\prime \prime}$ is a semifinite factor.

(ii) $\quad U_{c}^{y}(g) \cong \int_{S^{*}(y)}^{\oplus} d b U_{c}^{b}(g), g \in G$, is a factor decomposition of $U_{c}^{y}$; $U_{c}^{y}(G)^{\prime \prime} \cap U_{c}^{y}(G)^{\prime}$ is identified with $L^{\infty}\left(S^{*}(y)\right)$.

\section{§2. Borel Structure of $S^{*}$}

Let $S^{*}=\underset{y \in \Gamma^{(0)}}{\Perp} S^{*}(y)$ (disjoint union) and $p: S^{*} \rightarrow \Gamma^{(0)}$ be the canonical projection. In this section, we equip $S^{*}$ with a suitable Borel structure. 
Lemma 2.1. Let $Y$ be a Polish space and $X$ be an analytic Borel space. We assume that there are a Borel equivalence relation $R$ and an $R$-ergodic measure $\mu$ in $X$. Suppose that for each $x \in X$, a closed subset $F(x)$ of $Y$ is assigned and satisfies

(i) $\tilde{F} \equiv\{(x, y) ; x \in X, y \in F(x)\}$ is a Borel subset of $X \times Y$.

(ii) If $x \stackrel{R}{\sim} x^{\prime}\left(x, x^{\prime} \in X\right), F(x)=F\left(x^{\prime}\right)$.

Then there exists a $\mu$-negligible saturated set $N \subset X$ such that $F(x)=F\left(x^{\prime}\right)$ for all $x, x^{\prime} \in X \backslash N$.

Proof. Let $\left\{U_{i}\right\}_{i \geq 1}$ be a countable open base for $Y$. Let $A_{i}$ be the image of $F_{i} \equiv \tilde{F} \cap\left(X \times U_{i}\right)$ under the projection $X \times Y \rightarrow X$, which is a saturated set by (ii). Since $A_{i}$ is an analytic set as the image of analytic set and every analytic set is absolutely measurable, the ergodicity implies that either $\mu\left(A_{i}\right)=0$ or $\mu\left(X \backslash A_{i}\right)=0$. Now let

$$
N_{i}=\left\{\begin{array}{l}
A_{i} \text { if } \mu\left(A_{i}\right)=0, \\
X \backslash A_{i} \text { if } \mu\left(X \backslash A_{i}\right)=0,
\end{array}\right.
$$

and set $N=\bigcup_{i \geq 1} N_{i}$. By the construction, if $x, x^{\prime} \in X \backslash N$,

$$
\left\{y \in Y ;(x, y) \in F_{i}\right\} \neq \emptyset \Leftrightarrow\left\{y \in Y ;\left(x^{\prime}, y\right) \in F_{i}\right\} \neq \emptyset .
$$

Since $F(x)$ is closed, this implies that $F(x)=F\left(x^{\prime}\right)$.

\section{Lemma 2. 2.}

(i) $\tilde{S}=\left\{(x, g) \in \Gamma^{(0)} \times G ; g \in S(x)\right\}$ is a Borel set of $\Gamma^{(0)}$.

(ii) $S(s(\gamma))=S(r(\gamma))$ for $\gamma \in \Gamma$.

Proof. (i): Take a countable dense subset $\left\{g_{i}\right\}_{i \geq 1}$ of $G$. Since

$$
G \times G \ni\left(g, g^{\prime}\right) \mapsto c_{x}\left(g, g^{\prime}\right) / c_{x}\left(g^{\prime}, g\right)
$$

is continuous ([6] Prop. 1.5), $\tilde{S}=\bigcap_{i \geq 1}\left\{(x, g) ; c_{x}\left(g, g_{i}\right)=c_{x}\left(g_{i}, g\right)\right\}$ is a Borel set.

(ii): This follows from

$$
\frac{c_{y}\left(g, g^{\prime}\right)}{c_{x}\left(g^{\prime}, g\right)}=\frac{c\left(\gamma, g g^{\prime}\right)}{c\left(g g^{\prime}, \gamma\right)} \frac{c(g, \gamma)}{c(\gamma, g)} \frac{c\left(g^{\prime}, \gamma\right)}{c\left(\gamma, g^{\prime}\right)},
$$

which is an easy consequence of cocycle relation.

Due to above two lemmas, $S(x)$ is equal to a closed subgroup of 
$G$, say $S$, for $\mu$-a.e. $x \in \Gamma^{(0)}$. So, for the purpose of factor decomposition, we may suppose that $S(x)=S$ for all $x \in \Gamma^{(0)}$ (inessential reduction).

To define a Borel structure in $S^{*}$, we need a special class of sections of $S^{*} \rightarrow \Gamma^{(0)}$. Let $L^{\infty}(S, T)$ be a subset of $L^{\infty}(S)$, consisting of $T$-valued measurable function on $S$ and we give it weak* topology induced from $L^{\infty}(S)$. Then $L^{\infty}(S, T)$ is a Polish group by pointwise multiplication. Similarly $L^{\infty}(S \times S, T)$ is a Polish group. Define a continuous homomorphism $\delta ; L^{\infty}(S, T) \rightarrow L^{\infty}(S \times S, T)$ by $(\delta b)\left(g, g^{\prime}\right)=b(g) b\left(g^{\prime}\right) b\left(g g^{\prime}\right)^{-1}$. Then $\delta^{-1}(1)=$ the inverse image of $\delta$ at unit of $L^{\infty}(S \times S, T)$ is a closed subgroup of $L^{\infty}(S, T)$ and naturally identified with the dual group of $S$. Set

(19) $C=\left\{[c] \in L^{\infty}(S \times S, T) ; c\right.$ is a symmetric Borel 2-cocycle of $\left.S\right\}$.

Since symmetric cocycle is trivial and the image of $\delta$ is always symmetric, we have $C=\delta\left(L^{\infty}(S, \boldsymbol{T})\right)$. So $\delta$ induces a continuous isomorphism $\delta_{*}$ of $L^{\infty}(S, T) / \delta^{-1}(1)$ onto $C$, from which one sees that $C$ is a Borel subset of $L^{\infty}(S \times S, T)$ and $\delta_{*}$ is a Borel isomorphism (note that $L^{\infty}(S, \boldsymbol{T}) / \delta^{-1}(1)$ is a Polish group). Since the natural projection $L^{\infty}(S, \boldsymbol{T}) \rightarrow L^{\infty}(S, \boldsymbol{T}) / \delta^{-1}(1)$ has a Borel section ([1] Th. 3.4.1), $\delta$ also has a Borel section on C. Due to the definition of $S$, $c_{x}\left(g, g^{\prime}\right)$ is a symmetric Borel cocycle on $S$, and we have a Borel map $\Gamma^{(0)} \ni x \mapsto\left[c_{x}\right] \in C$. As a conclusion, we can find a Borel map $\beta: \Gamma^{(0)} \ni x \mapsto \beta_{x} \in L^{\infty}(S, T)$ such that $\delta\left(\beta_{x}\right)=\left[c_{x}\right]$. Since in the class $\beta_{x}$ there is one and only one Borel function $b_{x}$ on $S$ satisfying $b_{x}(g) b_{x}\left(g^{\prime}\right) b_{x}\left(g g^{\prime}\right)^{-1}=c_{x}\left(g, g^{\prime}\right)$ (cf [2] p. 308), we have proved the following :

Lemma 2.3. We can find a function $\Gamma^{(0)} \times S \ni(x, g) \mapsto b_{x}(g) \in \mathbb{T}$ such that

(i) for each $x \ni \Gamma^{(0)}, S \in g_{\mapsto} b_{x}(g)$ is a Borel function on $S$ and satisfies $b_{x}(g) b_{x}\left(g^{\prime}\right) b_{x}\left(g g^{\prime}\right)^{-1}=c_{x}\left(g, g^{\prime}\right), g, g^{\prime} \in S$.

(ii) $\Gamma^{(0)} \ni x \mapsto\left[b_{x}\right] \in L^{\infty}(S, T)$ is a Borel map.

Now we define a Borel structure in $S^{*}$. Take a function $b_{x}(g)$ satisfying conditions in Lemma 2.3. We have a bijection $\Gamma^{(0)} \times \widehat{S} \ni$ $(x, \chi) \mapsto\left(x, b_{x} \chi\right) \in S^{*}$, which transfers the Borel structure of $\Gamma^{(0)} \times \hat{S}$ into $S^{*}$. 
Lemma 2. 4. The above mentioned Borel structure of $S^{*}$ is independent of the choice of $b$.

Proof. Let $b_{x}^{\prime}(g)$ be another such function. By condition (i) in Lemma 2.3, we can find a function $\Gamma^{(0)} \ni x \mapsto \chi_{x} \in \hat{S}$ such that $b_{x}^{\prime}(g)=$ $\chi_{x}(g) b_{x}(g)$ and by (ii) in Lemma $2.3, \Gamma^{(0)} \ni x \mapsto\left[\chi_{x}\right] \in \delta^{-1}(1) \cong \hat{S}$ is a Borel map. So $\Gamma^{(0)} \times S \ni(x, g) \mapsto \chi_{x}(g)$ is a Borel function and this implies that two Borel structures coincide.

\section{§3. Factor Decomposition of $\mathscr{U}_{\text {。 }}$}

Take and fix a Haar measure $d s$ in $S$ and a Haar measure $d \chi$ in $S$ which are dually related, i. e., $\int d s \int d \chi f(\chi) \chi(s)=f(1)$ for $f \in C_{c}(S)$. $d s$ determines a Hilbert space $\mathfrak{S}_{b}$ for each $b \in S^{*}$ (see near (12)) and $d \chi$ is transformed to an $\hat{S}$-invariant measure $\lambda^{x}$ of $S^{*}(x)$ for each $x \in \Gamma^{(0)}$. Then $\left\{\lambda^{x}\right\}_{x \in \Gamma^{(0)}}$ forms a Borel field of measures and $\int_{\Gamma^{(0)}} \mu(d x) \lambda^{x}(d b)$ defines a measure $\hat{\mu}$ in $S^{*}$. Since $d s$ and $d \chi$ are dually related, $\xi \mapsto \int_{S^{*}}^{\oplus} \hat{\mu}(d b) \xi_{b}$ gives rise to a unitary map

$$
L^{2}(\Gamma, m) \Im \int_{S^{*}}^{\oplus} \hat{\mu}(d b) \mathfrak{S}_{b} .
$$

Let $T \in \mathfrak{Y}_{c}^{\prime} \cap \mathfrak{U}_{c}^{\prime \prime}$. Then, due to Lemma 1.2 and Th. II. 3. 1 in [4], we can find a measurable family of operators $\left\{T^{y}\right\}_{y \in \Gamma^{(0)}}, T^{y} \in \mathscr{B}\left(L^{2}\left(\Gamma^{y}, \nu^{y}\right)\right)$ such that

$$
T=\int^{\oplus} \mu(d y) T^{y} \text { and } T^{y} \in U_{c}^{y}(G)^{\prime \prime} \cap U_{c}^{y}(G)^{\prime} .
$$

By Lemma 1.3, above isomorphism (20) transforms $\int^{\oplus} \mu(d y) T^{y}$ into a diagonalizable operator in $\int_{S^{*}}^{\oplus} \hat{\mu}(d b) \mathfrak{S}_{b}$. Thus $\mathfrak{U}_{c}^{\prime \prime} \cap \mathfrak{H}_{c}^{\prime}$ is identified with a closed subalgebra of $L^{\infty}\left(S^{*}, \hat{\mu}\right)$. Conversely, let $F \in L^{\infty}\left(S^{*}, \hat{\mu}\right)$. If we regard $F$ as $\int^{\oplus} \mu(d y) \quad F^{y}$ with $F^{y} \in U_{c}^{y}(G)^{\prime \prime} \cap U_{c}^{y}(G)^{\prime}$, then $F^{y}$ commutes with $R^{y}\left(\xi_{n}\right)(n \geq 1)$ and therefore $F$ commutes with $\mathfrak{U}_{c}^{\prime}$. Thus $F \in \mathfrak{R}_{c}^{\prime \prime}$.

Let us seek the condition when $F$ belongs to $\mathfrak{X}_{c}^{\prime}$. We begin with the construction of an action of $\Gamma$ on $S^{*}$. Let $\gamma \in \Gamma$ and $b \in S(r(\gamma))$ and define $b \gamma \in S^{*}(s(\gamma))$ by 


$$
(b \gamma)(g)=b(g) c(\gamma, g) / c(g, \gamma), g \in S \text {. }
$$

Then

$$
b\left(\gamma_{1} \gamma_{2}\right)=\left(b \gamma_{1}\right) \gamma_{2}, \text { for }\left(\gamma_{1}, \gamma_{2}\right) \in \Gamma^{(2)}, b \in S^{*}(r(\gamma)),
$$

$\hat{\Gamma} \equiv\left\{(b, \gamma) \in S^{*} \times \Gamma ; b \in S^{*}(r(\gamma))\right\}$ is a Borel set of $\Gamma \times S^{*}$, and $\hat{\Gamma} \ni$ $(b, \gamma) \mapsto b \gamma \in S^{*}$ is a Borel map on this set. So we can makc $\hat{\Gamma}$ into a groupoid: The unit space of $\hat{\Gamma}$ is $S^{*}$ and for $(b, \gamma) \in \hat{\Gamma}, r(b, \gamma)=b$, $s(b, \gamma)=b \gamma . \quad(b, \gamma)$ and $\left(b^{\prime}, \gamma^{\prime}\right) \in \hat{\Gamma}$ are composable if and only if $s(b, \gamma)=r\left(b^{\prime}, \gamma^{\prime}\right)$ and the composition $(b, \gamma)\left(b^{\prime}, \gamma^{\prime}\right)$ is given by ' $\left(b, \gamma \gamma^{\prime}\right)$. Since $\hat{\Gamma}^{b}$ is identified with $\Gamma^{p(b)}, \nu^{p(b)}$ determines a measure $\hat{\nu}^{b}$ in $\hat{\Gamma}^{b}$ and $\left\{\hat{\nu}^{b}\right\}_{b \in S^{*}}$ forms a transverse function for $\hat{\Gamma}$. The pair $(\hat{\mu}, \hat{\nu})$ gives a transverse measure of $\hat{\Gamma}$. We define a unitary map $U_{c}^{b}(\gamma)$ from $\mathfrak{S}_{b}$ onto $\mathfrak{S}_{b_{\gamma}{ }^{-1}}$ by

$$
\left(U_{c}^{b}(\gamma) \xi\right)\left(\gamma^{\prime}\right)=c\left(\gamma, \gamma^{-1} \gamma^{\prime}\right) \xi\left(\gamma^{-1} \gamma^{\prime}\right), \quad \xi \in \mathscr{S}_{b} .
$$

Now the following lemma is immediate.

Lemma 3.1. According to decompositions

$$
L^{2}\left(\Gamma^{x}, \nu^{x}\right) \cong \int_{S^{*}(x)}^{\oplus} \lambda^{x}(d b) \mathscr{\aleph}_{b}
$$

and

$$
L^{2}\left(\Gamma^{y}, \nu^{y}\right) \cong \int_{S^{\bullet}(y)}^{\oplus} \lambda^{y}(d b) \mathscr{Q}_{b}, \quad U_{c}(\gamma): L^{2}\left(\Gamma^{x}, \nu^{x}\right) \rightarrow L^{2}\left(\Gamma^{y}, \nu^{y}\right)
$$

is decomposed to $\int_{s^{\bullet}(x)}^{\oplus} \lambda^{x}(d b) U_{c}^{b}(\gamma)$.

Lemma 3.2. Let $L^{\infty}\left(S^{*} / \Gamma\right)=\left\{F \in L^{\infty}\left(S^{*}, \hat{\mu}\right) ; F(b)=F(b \gamma)\right.$ for $\hat{m}-$ a.e. $(b, \gamma) \in \hat{\Gamma}$, where $\hat{m}=\hat{\mu} \circ \hat{\nu}$ is a $\sigma$-finite measure in $\hat{\Gamma}$. Then

(i ) $L^{\infty}\left(S^{*} / \Gamma\right)$ is a weakly closed ${ }^{*}$-subalgebra of $L^{\infty}\left(S^{*}, \hat{\mu}\right)$.

(ii) For each class $F$ in $L^{\infty}\left(S^{*} / \Gamma\right)$, we can find a Borel function $f$ on $S^{*}$ such that $f$ is a representative of $F$ and $f(b)=f(b \gamma)$ for all $(b, \gamma) \in \hat{\Gamma}$.

Proof. (i) is immediate. (ii) follows from the proof of [3] Prop. II. 8.

Combining these lemmas with Lemma 1.1, we obtain

Corollary 3. 3. $F \in L^{\infty}\left(S^{*}, \hat{\mu}\right)$ belongs to $\mathfrak{U}_{c}^{\prime \prime} \cap \mathfrak{Y}_{c}^{\prime}$ if and only if $F(b)=$ $F(b \gamma)$ for $\hat{m}-a . e .(b, \gamma) \in \hat{\Gamma}$. 
Let us introduce an equivalence relation $\sim$ in $S^{*}$ by $b \sim b^{\prime} \Leftrightarrow b^{\prime}=b \gamma$ for some $r \in \Gamma$.

Corollary 3.4. Ergodic decomposition of $\left(S^{*}, \hat{\mu}, \sim\right)$ gives a factor decomposition of $\mathfrak{\mathfrak { A }}_{c}^{\prime}$.

\section{§4. Ergodic Decomposition of $S^{*}$}

In this section we assume that $S$ is a discrete subgroup of $G$. In that case, we can go further into the ergodic decomposition of $S^{*}$. We begin with the selection of certain subgroup of $S$. For $g \in G$, $C_{\mathrm{g}}$ is, by definition, the set of all Borel functions $f$ on $\Gamma^{(0)}$ such that

$$
f(r(\gamma))=f(s(\gamma)) c(g, \gamma) / c(\gamma, g) \text { for all } \gamma \in \Gamma \text {. }
$$

We identify two $\mu$-a.e. equal functions in $C_{\mathrm{g}}$.

Lemma 4. 1. For $f \in C_{g},|f(x)|$ is constant for $\mu$-a.e. $x \in \Gamma^{(0)}$ and two functions in $C_{g}$ is proportional (up to $\mu$-negligible set).

Proof. An immediate consequence of the ergodicity of $\mu$.

Lemma 4. 2. Set $\Sigma=\left\{g \in G ; C_{g} \neq\{0\}\right\}$. Then

( $i$ ) there exists a $\mu$-negligible saturated Borel set $N$ such that

$$
\Sigma \subset S(x) \text { for } x \in \Gamma^{(0)} \backslash N \text {. }
$$

(ii) $\Sigma$ is a subgroup of $G$.

Pronf. (i) Take a countable dense set $\left\{g_{i}\right\}_{i \geq 1}$ of $\Sigma$ and let $f_{i}$ be a non-trivial function in $C_{g_{i}}(i \geq 1)$. Then the saturated Borel set $N=\left\{x \in \Gamma^{(0)} ; f_{i}(x)=0\right.$ for some $\left.i \geq 1\right\}$ is $\mu$-negligible, and we have $\left\{g_{i}\right\}_{i \geq 1} \subset S(x)$ for $x \in \Gamma^{(0)} \backslash N$. Since $S(x)$ is closed, this proves (i). (ii) Let $g_{1}, g_{2} \in \Sigma$ and take non-trivial $f_{i} \in C_{g_{i}}(i=1,2)$. Set $f(x)=$ $f_{1}(x) f_{2}(x)^{-1} c_{x}\left(g_{1}, g_{2}^{-1}\right)^{-1} c_{x}\left(g_{2}, g_{2}^{-1}\right)\left(f_{2}(x)^{-1}\right.$ is defined to be zero if $f_{2}(x)$ $=0)$. Due to the cocycle relation, one sees that $f \in C_{g_{1} g_{2}^{-1}}$, which implies $g_{1} g_{2}^{-1} \in \Sigma$. Thus $\Sigma$ is a subgroup of $G$. 


\section{Definition 4. 3.}

$\Omega \equiv\left\{\omega ; \omega\right.$ is a function from $\Sigma$ into $L^{\infty}\left(\Gamma^{(0)}, \mathbb{T}\right)$ such that $\omega_{g} \in C_{g}$ and $\omega_{g_{1}}(x) \omega_{g_{2}}(x)=c_{x}\left(g_{1}, g_{2}\right) \omega_{g_{1} g_{2}}(x)$ for $\mu$-a. e. $x \in \Gamma^{(0)}$ (coboundary condition)\}.

Let us check that $\Omega$ is not void. Due to Lemma 4.1, we can find a function on $\Sigma \times \Gamma^{(0)},(g, x) \mapsto f_{g}(x)$ with the property, $f_{g} \in C_{g}$ and $\left|f_{g}(x)\right|=1$ for $\mu$-a. e. $x \in \Gamma^{(0)}$. For $g_{1}, g_{2} \in \Sigma$,

$$
x \mapsto f_{g_{1}}(x) f_{g_{2}}(x) f_{g_{1} g_{2}}(x)^{-1} c_{x}\left(g_{1}, g_{2}\right)^{-1}
$$

is a $\mu$-measurable function and by (18) it is constant on canonical equivalence class. So by the ergodicity of $\mu$, there is a unique $a\left(g_{1}, g_{2}\right)$ $\in \boldsymbol{T}$ such that

$$
f_{g_{1}}(x) f_{g_{2}}(x)=f_{g_{1} g_{2}}(x) c_{x}\left(g_{1}, g_{2}\right) a\left(g_{1}, g_{2}\right) \text { for } \mu \text {-a. e. } x \in \Gamma^{(0)} \text {, }
$$

and from this relation $a\left(g_{1}, g_{2}\right)$ is a symmetric cocycle on $\Sigma$. So we can find a function b: $\Sigma \rightarrow \mathbb{T}$ such that $a\left(g_{1}, g_{2}\right)=b\left(g_{1} g_{2}\right) b\left(g_{1}\right)^{-1} b\left(g_{2}\right)^{-1}$. Replacing $f_{g}$ with $b(g) f_{g}$, we may assume that $a\left(g_{1}, g_{2}\right) \equiv 1$. In other words, $g \mapsto\left[f_{g}\right] \in L^{\infty}\left(\Gamma^{(0)}, \mathbb{T}^{\prime}\right)$ is in $\Omega$ 。

Let $\omega, \omega^{\prime} \in \Omega$. By equivariance condition, there is a uniquely defined $T$-valued function $\chi$ on $\Sigma$ satisfying $\omega_{g}^{\prime}=\chi(g) \omega_{g}, g \in \Sigma$. Then, by coboundary condition, $\chi$ is a character on $\Sigma$. Conversely for each $\chi \in \hat{\Sigma}$ and each $\omega \in \Omega, g \mapsto \chi(g) \omega_{g}$ defines an element $\chi \omega$ in $\Omega . \hat{\Sigma} \times \Omega$ $\ni(\chi, \omega) \mapsto \chi \omega \in \Omega$ is an action of $\hat{\Sigma}$ on $\Omega$, and with respect to which, $\Omega$ is a $\hat{\Sigma}$-principal homogeneous space. Since $\hat{\Sigma}$ is a compact abelian group, $\Omega$ has a unique $\hat{\Sigma}$-invariant probability measure $d \omega$ 。 In the rest of this section, we show that $L^{\infty}\left(S^{*} / \Gamma\right)$ is isomorphic to $L^{\infty}(\Omega, d \omega)$.

Let $\mathscr{C}$ be the set of measurable functions $\varphi$ on $\Gamma^{(0)} \times \Sigma$ such that

$$
\begin{aligned}
& \varphi(r(\gamma), g)=\varphi(s(\gamma), g) c(\gamma, g) / c(g, \gamma) \\
& \text { for } g \in \Sigma \text { and } \gamma \in \Gamma,
\end{aligned}
$$

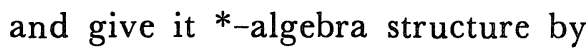

$$
\begin{aligned}
& \left(\varphi_{1} \varphi_{2}\right)(x, g)=\sum_{g^{\prime} \in \Sigma} \varphi_{1}\left(x, g^{\prime}\right) \varphi_{2}\left(x, g^{\prime-1} g\right) c_{x}\left(g^{\prime}, g^{\prime-1} g\right) \\
& \varphi^{*}(x, g)=\overline{\varphi\left(x, g^{-1}\right) c_{x}\left(g, g^{-1}\right)} .
\end{aligned}
$$

Further, inner product in $\mathscr{C}$ is introduced as 


$$
\left(\varphi_{1} \mid \varphi_{2}\right)=\sum_{g \in \Sigma} \overline{\varphi_{1}(x, g)} \varphi_{2}(x, g) .
$$

Here we must comment on the meaning of the right hand side. Since $x \mapsto \overline{\varphi_{1}(x, g)} \varphi_{2}(x, g)$ is constant on canonical equivalence class, it is constant, say $a_{g}$, for $\mu$-a. e. $x \in \Gamma^{(0)}$. The summation in (31) is, then, defined to be $\sum_{g \in \Sigma} a_{g}$. With all these structures, $\mathscr{C}$ becomes a commutative Hilbert algebra. A representation $\Phi$ of $\mathscr{C}$ on $L^{2}\left(\Gamma^{(0)}, \mu\right) \otimes$ $\ell^{2}(S)$ is defined by

$$
(\Phi(\varphi) \xi)(x, g)=\sum_{g^{\prime} \in \Sigma} \varphi\left(x, g^{\prime}\right) \xi\left(x, g^{-1} g\right) c_{x}\left(g^{\prime}, g^{\prime-1} g\right), x \in \Gamma^{(0)}, g \in S .
$$

Lemma 4.4. $\Phi$ is extended continuously to an isomorphism of $\mathscr{C}^{\prime \prime}$ onto $\Phi(\mathscr{C})^{\prime \prime}$.

Proof. See the argument before Theorem 1 in [9].

To relate $\Phi(\mathscr{C})^{\prime \prime}$ with $L^{\infty}\left(S^{*} / \Gamma\right)$, we use a partial Fourier transform. Let $\xi$ be a support-finite function on $S$ and $y \in \Gamma^{(0)}$. Set

$$
\hat{\xi}(b)=\sum_{g \in S} \xi(g) b(g) \text { for } b \in S^{*}(y) .
$$

Then $\hat{\xi}$ is in $L^{2}\left(S^{*}(y), \lambda^{y}\right)$ and $\xi \mapsto \hat{\xi}$ is extended to a unitary map of $\ell^{2}(S)$ to $L^{2}\left(S^{*}(y), \lambda^{y}\right)$, which is also denoted by ^ (this is essentially Fourier transform of $S$ ). Now let $\left\{\xi_{x}\right\}_{x \in \Gamma^{(0)}}$ be a family of vectors in $\ell^{2}(S)$. Then it can be easily checked that $\left\{\xi_{x}\right\}_{x \in \Gamma^{(0)}}$ is $\mu$-measurable if only if $\left\{\hat{\xi}_{x}\right\}_{x \in \Gamma^{(0)}}$ is $\mu$-measurable. Then a unitary map $V$ of $L^{2}\left(\Gamma^{(0)}, \mu\right) \otimes /^{2}(S)$ to $L^{2}\left(S^{*}, \hat{\mu}\right)=\int^{\oplus} \mu(d x) L^{2}\left(S^{*}(x), \lambda^{x}\right)$ is defined by

$$
V \xi=\int^{\oplus} \mu(d x) \hat{\xi}_{x} \quad \text { if } \quad \xi=\int^{\oplus} \mu(d x) \xi_{x} \text { with } \xi_{x} \in \ell^{2}(S) .
$$

\section{Lemma 4.5.}

$$
V^{*} L^{\infty}\left(S^{*} / \Gamma\right) V=\Phi(\mathscr{C})^{\prime \prime}
$$

Proof. Let $\varphi \in \mathscr{C}$. A direct computation shows that $V \Phi(\varphi) V^{*}$ is a multiplication operator by

$$
\hat{\varphi}(b) \equiv \sum_{g \in \Sigma} \varphi(p(b), g) b(g), b \in S^{*},
$$

and $\hat{\varphi}$ is constant on equivalence class of $\sim$ (due to (27)). Thus $V \Phi(\mathscr{C})^{\prime \prime} V^{*} \subset L^{\infty}\left(S^{*} / \Gamma\right)$. Conversely let $F \in L^{\infty}\left(S^{*} / \Gamma\right)$. According to 
Lemma 3.2 , we may assume that $F$ is represented by a $\hat{\mu}$-measurable function $f$ on $S^{*}$ satisfying

$$
f(b \gamma)=f(b) \text { for all }(b, \gamma) \in \hat{\Gamma} \text {. }
$$

Let $\xi \in L^{2}\left(\Gamma^{(0)}, \mu\right) \otimes \ell^{2}(S)$ be $S$-support finite; there is a finite set $F$ of $S$ such that if $g \notin F, \xi(x, g)=0$ for $\mu$-a. e. $x \in \Gamma^{(0)}$. By a direct computation, we have

$$
\left(V^{*} F V \xi\right)(x, g)=\sum_{g^{\prime} \in S} \varphi\left(x, g^{\prime}\right) \xi\left(x, g^{\prime-1} g\right) c_{x}\left(g^{\prime}, g^{\prime-1} g\right),
$$

$(x, g) \in \Gamma^{(0)} \times S$, where a measurable function $\varphi$ on $\Gamma^{(0)} \times S$ is defined by

$$
\varphi(x, g)=\int_{S^{*}(x)} \lambda^{x}(d b) f(b) b(g)^{-1}
$$

Due to (35), $\varphi$ satisfies

$$
\varphi(r(\gamma), g)=\varphi(s(\gamma), g) c(\gamma, g) / c(g, \gamma)
$$

and then, by the definition of $\Sigma, \varphi$ vanishes outside of $\Sigma$. Since such a function is approximated by elements in $\mathscr{C}$ (cf. arguments in the proof of [9] Theorem 1), we deduce that $V^{*} F V \in \Phi(\mathscr{C})^{\prime \prime}$.

Now we relate $\mathscr{C}^{\prime \prime}$ with $L^{\infty}(\Omega)$. This is also achieved by Fourier transform. Let $\varphi \in \mathscr{C}$ and define a function $W \varphi$ on $\Omega$ by

$$
W \varphi(\omega)=\sum_{g \in \Sigma} \varphi(x, g) \omega_{g}(x)
$$

where $\varphi(x, g) \omega_{g}(x)$ is constant for $\mu-a . e . x$ and the summation is taken over these constants. Since $\varphi$ has an $S$-finite support, the summation in (40) is finite and $W \varphi$ is in $L^{\infty}(\Omega) \subset L^{2}(\Omega) . \quad \varphi \mapsto W \varphi$ is extended to a unitary map from $L^{2}(\mathscr{C})$ to $L^{2}(\Omega)$, which is also denoted by $W$.

\section{Lemma 4.6.}

$$
W^{*} L^{\infty}(\Omega) W=\mathscr{C}^{\prime \prime}
$$

Proof. By a direct computation we have

$$
\left(W\left(\varphi_{1} \varphi_{2}\right)\right)(\omega)=\left(W \varphi_{1}\right)(\omega)\left(W \varphi_{2}\right)(\omega),
$$

$\omega \in \Omega, \varphi_{1}, \varphi_{2} \in \mathscr{C}$, and we can apply Stone-Weierstrass theorem to obtain the assertion.

Definition 4.7. Set $D=\{W \varphi ; \varphi \in \mathscr{C}\} . D$ is a dense $*$-subalgebra 
of $L^{\infty}(\Omega)$. We define a linear map $\tau$ of $D$ into $L^{\infty}\left(S^{*}, \hat{\mu}\right)$ by

$$
\tau(\psi)(b)=\sum_{g \in \Sigma} \int_{\Omega} d \omega \psi(\omega) b(g) \omega_{g}(p(b))^{-1}, b \in S^{*} .
$$

Here we give comments on the integration in (41). Take any $\omega^{0} \in \Omega$ and a representative of $\omega^{0}$ by a function $\omega_{g}^{0}(x)$. Then $\int_{\Omega} d \omega \phi(\omega) \omega_{g}^{0}(x)^{-1}$ is defined to be $\int_{\widehat{\Sigma}} d \chi \psi(\chi \omega) \chi(g)^{-1} \omega_{g}^{0}(x)^{-1}$. Another choice of $\omega^{0}$ and $\omega_{g}^{0}(x)$ gives the same integration for $\mu$-a.e.x. Thus as an element of $L^{\infty}\left(\Gamma^{(0)}, \mu\right), x \mapsto \int_{\Omega} d \omega \psi(\omega) \omega_{g}(x)$ is well-defined. Furthermore if we express $\psi$ as $W \varphi(\varphi \in \mathscr{C})$, then $\varphi(x, g)=\int_{\Omega} d \omega \psi(\omega) \overline{\omega_{g}(x)}$ for $g \in \Sigma$ and for $\mu$-a.e. $x \in \Gamma^{(0)}$. So the summation in (41) is essentially finite and the right hand side of (42) gives a well-defined element in $L^{\infty}\left(S^{*}\right)$.

Theorem 4.8. $\tau$ is extended to a normal ${ }^{*}$-isomorphism of $L^{\infty}(\Omega)$ into $L^{\infty}\left(S^{*}\right)$ and $\tau\left(L^{\infty}(\Omega)\right)=L^{\infty}\left(S^{*} / \Gamma\right)$.

Proof. A computation shows that

$$
\tau(W \varphi)=V \Phi(\varphi) V^{*}, \text { for } \varphi \in \mathscr{C} .
$$

Now the assertion follows from Lemma 4.5 4.7.

Corollary 4.9. $\mathfrak{2}_{c}^{\prime \prime}$ is a factor if and only if $\Sigma=\{e\}$.

\section{§5. Factor Decomposition of $(\Gamma, c)$}

In this section $S$ is continued to be assumed discrete, and we work out a factor decomposition of $\mathfrak{A}_{c}$ in groupoid level, using the results of $\S 4$. To simplify the construction, we adopt another point of view for the description of cocycle regular representation.

Definition 5.1. Let $\Gamma$ be a Borel groupoid and let $B=\left\{B_{\gamma}\right\}_{\gamma \in \Gamma}$ be a Borel field of 1-dimentional Hilbert spaces over $\Gamma$ and suppose that

(i) multiplication $B_{\gamma_{1}} \otimes B_{\gamma_{2}} \simeq B_{\gamma_{1} \gamma_{2}}\left(\left(\gamma_{1}, \gamma_{2}\right) \in \Gamma^{(2)}\right)$ is given. It is associative and Borel in the following sense: Let $\xi_{1}, \xi_{2}, \xi$ be a Borel section of $B$, then $\left(\gamma_{1}, \gamma_{2}\right) \mapsto\left(\xi_{1}\left(\gamma_{1}\right) \xi_{2}\left(\gamma_{2}\right) \mid \xi\left(\gamma_{1} \gamma_{2}\right)\right)$ is Borel,

(ii) anti-unitary involution ${ }^{*} B_{\tau} \rightarrow B_{\gamma-1}$ is given. It transforms Borel sections to Borel sections and satisfies 
(iii) $\quad\left(\xi_{1} \xi_{2} \mid \xi\right)=\left(\xi_{2} \mid \xi_{1}^{*} \xi\right), \quad \xi_{1} \in B_{\gamma_{1}}, \quad \xi_{2} \in B_{r_{2}}, \quad \xi \in B_{r_{1} r_{2}}$,

(iv) if $\xi \in B_{x}\left(x \in \Gamma^{(0)}\right)$ and $\xi^{2}=\xi \neq 0$, then $(\xi \mid \xi)=1$.

We call such $B$ a groupoid ring.

Take $0 \neq \xi \in B_{x}\left(x \in \Gamma^{(0)}\right)$. Since $B_{x} \otimes B_{x} \cong B_{x}, \xi^{2}=z \xi$ with $0 \neq z \in C$. Replacing $\xi$ by $z^{-1} \xi$, we may assume that $\xi^{2}=\xi$. Then by (iv), $(\xi \mid \xi)=1$. Let $\eta \in B_{r}$ with $s(\gamma)=x$. By $B_{r} \otimes B_{x} \cong B_{r}$, we can find $\eta^{\prime} \in B_{r}$ such that $\eta=\eta^{\prime} \xi$ and then $\eta \xi=\eta^{\prime} \xi^{2}=\eta^{\prime} \xi=\xi$, i.e., $\xi$ is a right unit for $B_{r}$. Furthermore, as $\eta^{*} \eta \in B_{x}$ and $\left(\eta^{*} \eta \mid \xi\right)=(\eta \mid \eta \xi)=(\eta \mid \eta)$, we conclude that $\eta^{*} \eta=(\eta \mid \eta) \xi$. As a corollary of this fact, $\{\xi \in B ;(\xi \mid \xi)$ $=1\}$ is closed under multiplication. Above arguments also show that there is a Borel section $\sigma: \Gamma \rightarrow B$ such that

$$
\begin{aligned}
& (\sigma(\gamma) \mid \sigma(\gamma))=1 \text { for } \gamma \in \Gamma, \\
& \sigma(x)^{2}=\sigma(x) \text { if } x \in \Gamma^{(0)} .
\end{aligned}
$$

Associated with $\sigma$, we define a $T$-valued Borel 2-cocycle $c$ of $\Gamma$ by

$$
\sigma\left(\gamma_{1}\right) \sigma\left(\gamma_{2}\right)=c\left(\gamma_{1}, \gamma_{2}\right) \sigma\left(\gamma_{1} \gamma_{2}\right),\left(\gamma_{1}, \gamma_{2}\right) \in \Gamma^{(2)} \text {. }
$$

Due to (44), $c$ is normalized, i. e., $c(\gamma, s(\gamma))=c(r(\gamma), \gamma)=1$ for $\gamma \in \Gamma$. If we change $\sigma$ (under the condition that it satisfies (43) and (44)), $c$ is changed to a cohomologous one. In this way, groupoid ring $B$ determines an element in the Borel 2-cohomology group $H^{2}(\Gamma, T)$. Conversely, for any normalized Borel cocycle $c$, a groupoid ring structure is defined in the trivial bundle $B=\Gamma \times C$ by

$$
\begin{aligned}
& (\gamma, z)\left(\gamma^{\prime}, z^{\prime}\right)=\left(r \gamma^{\prime}, z z^{\prime} c\left(\gamma, \gamma^{\prime}\right)\right) \\
& (\gamma, z)^{*}=\left(\gamma^{-1}, \overline{z c\left(\gamma^{-1}, \gamma\right)}\right) \\
& ((\gamma, z) \mid(\gamma, z))=|z|^{2} .
\end{aligned}
$$

If we change $c$ by a coboundary, the groupoid ring obtained in this way is changed to an isomorphic one. So we have proved

Proposition 5.2. There is a 1-1 correspondence between isomorphism class of groupoid ring over $\Gamma$ and $\boldsymbol{T}$-valued cohomology class of $\Gamma$.

Now we can rewrite various objects related with a cocycle $c$, in terms of the corresponding groupoid ring $B$. For example $\mathfrak{A}_{c}$ is 
realized as a set $\mathfrak{A}$ of Borel sections of $B$. Its Hilbert algebra structure is described as

$$
\begin{aligned}
& \left(\xi_{1} \xi_{2}\right)(\gamma)=\int \nu^{r(r)}\left(d \gamma^{\prime}\right) \xi_{1}\left(\gamma^{\prime}\right) \xi_{2}\left(\gamma^{\prime-1} \gamma\right), \xi_{1}, \xi_{2} \in \mathfrak{A}, \gamma \in \Gamma . \\
& \xi^{*}(\gamma)=\xi\left(\gamma^{-1}\right)^{*}, \xi \in \mathfrak{A}, \gamma \in \Gamma . \\
& \left(\xi_{1} \mid \xi_{2}\right)=\int \mu(d y) \int \nu^{\nu}(d \gamma)\left(\xi_{1}(\gamma) \mid \xi_{2}(\gamma)\right),
\end{aligned}
$$

$\xi_{1}, \xi_{2} \in \mathfrak{A} . \quad C_{g}, g \in G$ (see $\S 4$ ) is also described by making use of $\mathrm{B}$. Let $B^{\mathrm{g}}$ be a Borel line bundle over $\Gamma^{(0)}$ defined by $B^{\mathrm{g}}=\underset{x \in \Gamma(0)}{\cup} B_{c_{x}(g)}$. Each $\gamma \in \Gamma$ gives rise to a linear map of $B_{s(\gamma)}^{g}$ into $B_{r(\gamma)}^{g}, B_{s(\gamma)}^{g} \ni \eta \mapsto \gamma \eta \equiv$ $\xi \eta \xi^{*} \in B_{r(\gamma)}^{g}$, where $\xi \in B_{\gamma}$ is a unit vector, and $B^{g}$ becomes a $\Gamma$-bundle. Now $C_{g}$ is identified with the set of Borel section $\xi$ of $B^{g}$ such that

$$
\xi(r(\gamma))=\gamma \xi(s(\gamma)), \quad \gamma \in \Gamma,
$$

and then $\Omega$ consists of sequences $\left\{\omega_{g}\right\}_{g \in \Sigma}\left(\Sigma=\left\{g \in G ; C_{g} \neq 0\right\}\right)$ such that

$$
\omega_{g} \in C_{g},\left\|\omega_{g}(x)\right\|=1,
$$

and

$$
\omega_{g_{1}}(x) \omega_{g_{2}}(x)=\omega_{g_{1} g_{2}}(x) \text { for } \mu-\text { a. e. } x \in \Gamma^{(0)} .
$$

Consider the quotient space $\Gamma / \Sigma$. It has a structure of analytic Borel groupoid induced from $\Gamma$. Let $\omega \in \Omega$ and define an action of $\Sigma$ on $B$ by

$$
g \xi=\omega_{g}(r(\gamma)) \xi \in B_{g_{\gamma}}, \quad \xi \in B_{\gamma} .
$$

Taking quotient, we have a groupoid ring $B^{\omega}$ over $\Gamma / \Sigma$ (cf. comments in Definition 4.7). Let $\mathfrak{A}_{\omega}$ be the Hilbert algebra associated with $B^{\omega}$. Note that each section of $B^{\omega}$ is identified with a $\Sigma$-equivariant section of B. Now we specify the Borel field structure for the collection of Hilbert algebras $\left\{\mathfrak{H}_{\omega}\right\}_{\omega \in \Omega}$. Let $\xi$ be a section in $\mathfrak{A}$ and suppose that $\{g \in \Sigma$; there exists $\gamma \in \Gamma$ such that $\xi(\gamma) \neq 0$ and $\xi(g \gamma) \neq 0\}$ is finite. Then we can form a family of vectors $\left\{\xi_{\omega}\right\}_{\omega \in \Omega} \in\left\{\mathfrak{R}_{\omega}\right\}_{\omega \in \Omega}$ by

$$
\xi_{\omega}(\gamma)=\sum_{g \in \Sigma} \omega_{g}(r(\gamma)) \xi\left(g^{-1} \gamma\right), \quad \gamma \in \Gamma .
$$

Such families for various $\xi$, give a Borel field structure and, from the results in $\S 4$, we obtain

\section{Theorem 5.3.}

(i) For each $\omega \in \Omega, \mathfrak{R}_{\omega}^{\prime \prime}$ is a factor. 
(ii) $\mathfrak{Q}^{\prime \prime} \cong \int_{\Omega}^{\oplus} d \omega \mathfrak{Y}_{\omega}^{\prime \prime}$ is a central factor decomposition of $\mathfrak{X}^{\prime \prime}\left(\mathfrak{X}^{\prime \prime} \cap \mathfrak{X}^{\prime}\right.$ corresponds to $\left.L^{\infty}(\Omega, d \omega)\right)$.

\section{§ 6. Example}

Let $\lambda_{i}(i=1,2)$ be a real number satisfying $1^{\circ} \lambda_{i} \notin Q \pi$ and $2^{\circ}$ there are integers $m_{1}, m_{2}, m$ such that $\lambda_{1} m_{1}+\lambda_{2} m_{2}=2 \pi m$ with $\left(m_{1}, m_{2}, m\right)=1$ (relatively prime), and define an action $\varphi$ of $\boldsymbol{Z}^{2}$ on $\boldsymbol{T}$ by

$$
\varphi_{\left(n_{1}, n_{2}\right)}(z)=e^{i\left(\lambda_{1} n_{1}+\lambda_{2} n_{2}\right)} z, \quad z \in \boldsymbol{T} .
$$

We construct a groupoid $\Gamma=Z^{2} \times \boldsymbol{T}$ by semi-direct product using $\varphi$, which is ergodic if we give a Haar measure to $\boldsymbol{T}$. Note that

$$
s\left(n_{1}, n_{2}, z\right)=z, \quad r\left(n_{1}, n_{2}, z\right)=\varphi_{\left(n_{1}, n_{2}\right)}(z),
$$

and

$$
\left(n_{1}, n_{2}, z\right)\left(n_{1}^{\prime}, n_{2}^{\prime}, z^{\prime}\right)=\left(n_{1}+n_{1}^{\prime}, n_{2}+n_{2}^{\prime}, z^{\prime}\right) .
$$

The stabilizer of $\Gamma$ at $z$ is $\left\{\left(m_{1} n, m_{2} n, z\right) \in \Gamma ; n \in Z\right\}$ and we can define isomorphism $\iota_{z}: Z \rightarrow \Gamma^{z}$ by $\iota_{z}(n)=\left(m_{1} n, m_{2} n, z\right) . \quad\left\{\ell_{z}\right\}_{z \in T}$ satisfies the conditions $1^{\circ}, 2^{\circ}$ in $\S 1$. For $\alpha \in \boldsymbol{R}$, let $c$ be a cocycle of $\Gamma$ given by

$$
c\left(n_{1}, n_{2}, z ; n_{1}^{\prime}, n_{2}^{\prime}, z^{\prime}\right)=e^{i \alpha / 2\left(n_{1} n_{2}^{\prime}-n_{1}^{\prime} n_{2}\right)} \text {. }
$$

Let us find out $\Sigma$. For $n \in Z(=G)$, the condition (25) is expressed in this example as

$$
f\left(\mathrm{e}^{i\left(\lambda_{1} n_{1}+\lambda_{2} n_{2}\right)} z\right)=\mathrm{e}^{-i \alpha n\left(m_{1} n_{2}-m_{2} n_{1}\right)} f(z),
$$

for $n_{1}, n_{2} \in \boldsymbol{Z}$ and for $a_{\text {. e. }} z \in \boldsymbol{T}$. Using Fourier expansion $f(z)=\sum_{k \in \boldsymbol{Z}} f_{k} z^{k}$, we have $f \neq 0$ if and only if the condition

(*) $\exists \mathrm{k} \in \boldsymbol{Z}$ such that $\mathrm{k} \lambda_{1}-\alpha m_{2} n \in 2 \pi \boldsymbol{Z}$, $k \lambda_{2}+\alpha m_{1} n \in 2 \pi Z$,

is satisfied.

Case $\alpha \notin \boldsymbol{Q} \lambda_{1}+\boldsymbol{Q} \pi$.

There is no $n \in Z$ satisfying $\left(^{*}\right)$, i. e., $\Sigma=0$, and $c$-regular representation generates a factor.

Case $\alpha \in \boldsymbol{Q} \lambda_{1}+\boldsymbol{Q} \pi$.

We can choose integers $a, b, c$ such that $\alpha a=\lambda_{1} b+2 \pi c$ with $(a, b, c)=1$. 
Then some computations show that $n \in \boldsymbol{Z}$ satisfies $\left(^{*}\right)$ if and only if it is an integer multiple of $a /\left(m_{2}, b m+c m_{1}, a\right)$, i. e., $\Sigma=a /\left(m_{2}, b m\right.$ $\left.+c m_{1}, a\right) \boldsymbol{Z}$. In this case, $c$-regular representation has a factor decomposition parametrized by $\hat{\Sigma} \cong \boldsymbol{T}$.

\section{References}

[1] W. Arveson, An Invitation to $C^{*}$-Algebras, Springer, 1976.

[2] L. Baggett and A. Kleppner, Multiplier representations of abelian groups, J.Funct. Analysis, 14(1973), 299-324.

[3] A. Connes, Sur la theorie non commutative de l'integration, Lect. Notes in Math., 725 (1979), 19-143.

[4] J. Dixmier, Von Neumann Algebras, North-Holland, 1981.

[5] P. Hahn, The regular representations of measure groupoids, Trans. Amer. Math. Soc., 242(1978), 35-72.

[6] A. Kleppner, Multipliers on Abelian Groups, Math. Ann., 158(1965), 11-34.

[7] , Non type I multiplier representations of abelian groups, Technical Report, Univ. of Maryland, 1975.

[8] L. Pukanszky, Unitary representations of solvable Lie groups, Ann. Sci. Ecole Norm. Sup., 4(1971), 464-608.

[9] S. Yamagami, The type of regular representations of a certain transitive groupoid, J. Operator Theory, 14(1985), 249-261.

[10] C. E. Sutherland, A Borel parametrization of Polish groups, preprint. 
